\title{
Functionalization of Carbon Nanotubes Via Cleavable Bonds for Efficient Intracellular Delivery of siRNA and Potent Gene Silencing
}

\author{
Nadine Wong Shi Kam, Zhuang Liu and Hongjie Dai* \\ Department of Chemistry Stanford University, Stanford, CA 94305
}

\section{Supplementary Information with Supplementary Figures}

\section{Functionalization of SWNTs by phospholipids with PEG chains (PL-PEG). Hipco}

SWNTs were sonicated extensively (1-1.5h) in a solution of 1,2-Distearoyl-sn-Glycero-3Phosphoethanolamine-N-[Amino(Polyethylene Glycol)2000] (Avanti Polar Lipids). The phospholipid molecule contained a polyethylene glycol chain (45 units) terminated with either an amine group (PL-PEG-NH $\mathrm{N}_{2}$ ) or a maleimide group (PL-PEG-maleimide). The typical PL-PEG concentration used was in the range of $0.1-1 \mathrm{mg} / \mathrm{mL}$. The suspension was then filtered through a membrane filter (Whatman, pore size $100 \mathrm{~nm}$ ) to remove excess phospholipids, rinsed thoroughly with $\mathrm{H}_{2} \mathrm{O}$ and re-suspended in either $\mathrm{H}_{2} \mathrm{O}$ or buffer. The suspension was centrifuged at 24,000 g for $6 \mathrm{~h}$ to remove impurities and large nanotubes bundles that aggregated as the sediment. The supernatant was collected and re-centrifuged under similar conditions, and the sediment was again discarded. The nanotubes present in the supernatant (after the two rounds of centrifugation) were short with tube lengths mainly in the range of $50-300 \mathrm{~nm}$ as revealed by AFM imaging (S_Figure 1). Both AFM imaging and UV-vis-NIR (Fig. 1b in main text) spectroscopic characterization revealed high purity of SWNTs in the final solution of SWNTs. 
Preparation of 1-X (SWNT-PL-PEG-SS-X). 2.5 mg of sulfosuccinimidyl 6-(3'-[2pyridyldithio]-propionamido)hexanoate (sulfo-LC-SPDP, Pierce) was added to a solution of SWNTs functionalized with PL-PEG- $\mathrm{NH}_{2}$ in $50 \mathrm{mM}$ phosphate buffer (supplemented with $0.15 \mathrm{M} \mathrm{NaCl}, \mathrm{pH}=7.4)$. The mixture was allowed to react at room temperature for 1 h, after which the conjugate was centrifuged through a centrifugal filter with molecular weight cutoff of $100 \mathrm{kD}$ (Millipore) for 5 min twice to remove excess sulfo-LC-SPDP cross-linkers. The nanotube conjugates were then re-suspended in $\mathrm{H}_{2} \mathrm{O}$ for conjugation with thiolated biological molecules including SH-DNA or SH-siRNA. The DNA sequence used in the current work was fluorescently labeled Cy3-SH-( $\left.\mathrm{CH}_{2}\right)_{6}$ CATTCCGAGTGTCCA, and the siRNA was a double stranded with sense sequence 5'SH-( $\left.\mathrm{CH}_{2}\right)_{6}$ CUGGACUUCCAGAAGAACAdTdT-3' and antisense sequence 5'-dTdTGACCUGAAGGUCUUCUUGU-3' (no fluorescent labeling of the siRNA). The conjugation was allowed to last overnight and the resulting 1-DNA or 1-SiRNA solution was then ready for further experiments.

Preparation of 2-X (SWNT-PL-PEG-X). SWNTs dispersed in PL-PEG-maleimide in a phosphate buffer of $\mathrm{pH} \sim 8$ were mixed with either SH-DNA or SH-siRNA and allowed to react for $\sim 3-4 \mathrm{~h}$ at room temperature. The resulting 2-DNA or 2-SiRNA conjugates (containing no disulfide bonds) were used to compare with the 1-counterpart with disulfide linkage. 
Cellular Incubation for DNA and SiRNA transfection. HeLa cells were grown in Dulbecco's Modified Eagle’s Medium (DMEM) supplemented with 10\% fetal bovine serum (FBS) and 1\% penicillin-streptomycin (all reagents from Invitrogen). Cells were plated into chambered coverslides or 24 well plates $24 \mathrm{~h}$ prior to incubation with 1- or 2SWNT conjugates at a concentration of $\sim 8 \times 10^{4}$ cells per well.

1-DNA and 2-DNA incubation: The SWNT conjugate was added to the chambered cover-slides containing HeLa cells in the DMEM medium supplemented with FBS and pen-strep. The concentration of SWNT and DNA was 1.5-2 mg/L and 200 nM respectively and the concentration of serum in the incubation is determined to be $\sim 5 \%$. The cells were incubated for $\sim 3 \mathrm{~h}$ at $37^{\circ} \mathrm{C}$ and $5 \% \mathrm{CO}_{2}$. After incubation, the cells were thoroughly washed and observed by confocal microscopy either immediately or up to 24h later.

1-SiRNA and 2-SiRNA incubation: Cells were plated into 24 well plates or 4 well chamber microscope slides and incubated with 1- or 2-SiRNA ([SWNT] 1.5-2 $\mathrm{mg} / \mathrm{L}$ and [siRNA] 50 or $500 \mathrm{nM}$ ) for $\sim 24 \mathrm{~h}$ at $37^{\circ} \mathrm{C}$ in a $5 \% \mathrm{CO}_{2}$ environment. The serum concentration in the incubation medium is estimated to be $\sim 2-5 \%$. After incubation, the cells were washed and supplemented with fresh medium and allowed to incubate for an additional 24-72 $\mathrm{h}$ for RNAi to take effect. For confocal microscopy analysis, cells were fixed on chambered slides, and for flow cytometry analysis, cells were first trypsinized and subsequently fixed. Following cell fixation, cells were incubated with primary antibody to lamin (Abcam) and then with a fluorescently labeled secondary antibody (Aldrich). Confocal microscopy imaging and flow cytometry probed the fluorescence signals of the secondary antibody. 
Lipofectamine-SiRNA incubation:_Lipofectamine was purchased from Invitrogen and used according to the manufacturer's directions. Briefly lipofectamine was incubated with siRNA at room temperature for 15 min. The lipofectamine-siRNA mixture was then added to HeLa cells under the same conditions as above, with [lipofectamine] = 1mg/L and [siRNA] $=50$ or $500 \mathrm{nM}$ in the incubation. The cells were allowed to incubate as described above and observed either by confocal microscopy or flow cytometry.

Confocal microscopy. Adherent HeLa cells were imaged directly on the chambered cover-glass or glass slide using a Zeiss LSM 510 confocal microscope. For assessment of nuclear translocation of DNA, HeLa cells were co-stained with a blue nuclear dye, Draq 5 (Axxora) for $\sim 5 \mathrm{~min}$ prior to confocal analysis.

Cell Cytometry. After incubation in 1-DNA or 2-DNA, cells were washed and resuspended in ice-cold PBS supplemented with $2 \%$ propidium iodide (PI). The mean fluorescence was measured from 10,000 cells by flow cytometry (Becton Dickinson FACScan). Cells that were co-stained with propidium iodide (corresponding to naturally occurring dead cells) were excluded from the data analysis.

For RNAi assay, cells transfected by SiRNA via SWNT carriers or Lipofecamine were first trypsinized and subsequently fixed. Following cell fixation, cells were incubated with primary antibody to lamin (Abcam) and then with a fluorescently labeled secondary antibody (Aldrich). Flow cytometry was then used to measure the mean fluorescence of 5,000 -10000- cells. The fluorescence detected was from the FITC tag on the secondary 
antibody. The fluorescence measurement from fixed, untreated cells was also recorded. The percent silencing reported in Figure 3 was derived from the difference between the fluorescence intensities of the samples that were treated with siRNA and that of the control cells.

\section{SWNT-DNA conjugates characterization.}

UV-vis-NIR spectroscopy. UV-vis-NIR measurement was carried out using a Cary 6000i spectrophotometer. The distinct peaks in UV-vis-NIR spectrum for 1-DNA shown in Fig. 1c are characteristic of highly dispersed single-walled nanotubes in the suspension. The presence of the peak around $550 \mathrm{~nm}$ is due to the Cy3 label on the DNA strand. Following the combined sonication, filtration and centrifugation steps, the nanotubes in the suspension were mostly individualized or small bundles of SWNTs without large aggregates or impurities in the solution.

Atomic Force Microscopy (AFM). AFM analysis of the conjugates were carried out by dropping $\sim 20 \mu \mathrm{L}$ of SWNT suspension onto a silicon oxide substrate. The droplet was allowed to stand for $\sim 20$ min at room temperature after which the substrate was rinsed with $\mathrm{H}_{2} \mathrm{O}$, dried under $\mathrm{N}_{2}$ flow and used for AFM imaging.

Determination of number of DNA strands per nanotube. UV-vis-NIR and fluorescence spectroscopy were used to estimate the number of DNA strands conjugated to a SWNT. 1-DNA conjugates with various loadings of DNA onto the tubes were made by conjugating a stock solution of SWNT-PEG-PL with various concentrations of cy3 labeled SH-DNA. The concentration of SWNTs was $10 \mathrm{nM}$ (as determined by UV-visNIR absorbance spectrum of SWNTs at $808 \mathrm{~nm}$ with an extinction coefficient of $7.9 \mathrm{x}$ 
$10^{6} \mathrm{M}^{-1} \mathrm{~cm}^{-1}$, see ref. 3). The resulting conjugates were filtered by using a centrifugal filter with MWCO 30kD to remove excess unbound cy3-DNA. The filtration step was repeated twice and the filtrates for each concentration were pooled. A standard titration curve for the absorbance $(\lambda=550 \mathrm{~nm})$ cy3 labeled SH-DNA was determined and used to calculate the concentration of excess DNA in the filtrates from the various 1-DNA conjugates. The data is then used to estimate the loading of DNA molecules on a SWNT. Alternatively, experiments were carried out to use the fluorescence intensity of the cy3 label $\left(\lambda_{\text {excitation }}=545 \mathrm{~nm}\right.$ and $\left.\lambda_{\text {emmission }}=565 \mathrm{~nm}\right)$ as a measure of DNA molecules that were in excess in the solution after conjugation reactions to SWNTs. Both the absorbance and fluorescence measurements revealed that the loading of DNA for the 1DNA conjugate was about 20 DNA molecules per SWNT (average length 200nm) after conjugation reaction of $2 \mu \mathrm{M}$ DNA with 10nM of SWNT used for the experiments in Fig.2b-c. Note that negligible amount of excess DNA was found in the filtrate when the original concentration of DNA used in the conjugation mixture was below $100 \mathrm{nM}$ with the concentration of SWNTs being $\sim 10 \mathrm{nM}$.

\section{Stability of SWNTs functionalized by phospholipids (SWNT-PL). The typical}

SWNT-PL suspensions were originally made with SWNT concentration of $250 \mathrm{mg} / \mathrm{L}$ and the final concentration of short SWNT in the supernatant following the centrifugation steps was estimated to be $\sim 25 \mathrm{mg} / \mathrm{L}$. The aqueous stability of our SWNT-PL conjugates was assessed at high temperatures and in high salt buffer solutions. In comparison with SWNT functionalized with other surfactants (tween-20, sodium dodecyl sulfate (SDS), gum arabic), SWNT-PL were the only ones that remained stable both under high 
temperature $\left(80^{\circ} \mathrm{C}\right)$ and high salt environment $(0.2 \mathrm{M}$ phosphate buffer, $\mathrm{pH} 7.0)$ (see

S_Figure 2). The suspension of SWNT-PL conjugated with either DNA or siRNA was stable for weeks and even up to months when stored at $4{ }^{\circ} \mathrm{C}$ (S_Figure 2).

\section{Ex-vitro disulfide cleavage of 1-DNA by DTT and monitoring of the cleavage by}

fluorescence measurement. A solution of 1-DNA (SWNT-PL-PEG-SS-cy3DNA) (in $10 \mathrm{mM}$ phosphate buffer, $\mathrm{pH}=7.0$ ) was exposed to dithiothreol (DTT, $10 \mathrm{mM}$, Aldrich) and allowed to react for $2 \mathrm{~h}$ at room temperature. Following the reaction, the mixture was filtered twice through a centrifugal membrane filter (molecular weight cutoff 50KD) and re-suspended in $\mathrm{H}_{2} \mathrm{O}$. Control solutions of 1-DNA and 2-DNA not exposed to DTT as well as or 2-DNA treated with DTT (as described for 1-DNA) were also filtered twice and re-suspended in $\mathrm{H}_{2} \mathrm{O}$. Fluorescence measurements for cy3 were carried out using a Fluorolog 3 fluorimeter for all solutions to obtain Fig. 1c (excitation $=545 \mathrm{~nm}$ and emission $=565 \mathrm{~nm}$ ). The fluorescence levels were normalized for the 1-DNA and 2-DNA samples. Note that the fluorescence signal for 2-DNA was not significantly changed after DTT treatment since no cleavable disulfide bond exists for DNA-Cy3 conjugated to SWNTs via the maleimide covalent chemistry.

\section{Silencing of Firefly Luciferase expression by SWNT delivered SiRNA.}

We have also obtained excellent siRNA delivery and silencing result with siRNA against the firefly luciferase gene by using SWNT transporters. This combined with the Lamin A/C silencing result proves the generality of SWNT transporters for siRNA delivery (all via the disulfide attachment) and potent RNAi functionality. 
Expression of Luciferase plasmids in HeLa cells. This first step of our experiment was used to deliver plasmid DNA into cells. Plasmid DNAs (pDNA) encoding firefly luciferase (pGL3-Control, 5256 bpa) and renilla luciferase (pRL-TK, $4045 \mathrm{bpa}$ ) were obtained from Promega and used for the expression of luciferase inside cells. Luciferase expression was achieved by using Lipofectamine 2000 as the transfection agent for the plasmids according to the manufacturer's recommended procedure. Briefly, HeLa cells were plated in a 48-well plate ( 2 x $10^{4}$ cells/well) for $24 \mathrm{~h}$ until they reached 50\% confluency, after which the medium was replaced with DMEM without serum. The cells were co-transfected with two luciferase plasmids (firefly luciferase, pGL3-control and renilla luciferase, pRL-TK) in the presence of lipofectamine by adding $0.1 \mu \mathrm{g}$ of pGL3 and $0.1 \mu \mathrm{g}$ of pRL-TK to each well. The final volume of each well was adjusted to $250 \mu l$. After 4-5 hours of transfection, the cell medium was adjusted such that it contained $10 \%$ FBS.

Luciferase silencing. Our RNAi study was specifically aimed at targeting the firefly luciferase gene (pGL3) by the use of a double-stranded RNA with sense sequence (5'-SH-( $\left.\mathrm{CH}_{2}\right)_{6}$ CUUACGCUGAGUACUUCGAdTdT-3') and antisense sequence (sequence 5'-UCGAAGUACUCAGCGUAAGdTdT-3'). Lipofectamine-siRNA and SWNT-S-S-siRNA conjugates were prepared as described for the lamin study. The cells that were pre-transfected by the Luciferase plasmids as described above were exposed to control siRNA (free siRNA), lipofectamine-siRNA and SWNT-PL-PEG-SS-siRNA respectively for $12 \mathrm{~h}$. The cells were then washed and allowed to incubate for an additional $36 \mathrm{~h}$ in fresh medium. The Luciferase expression level was then investigated by using a dual Luciferase assay kit that contains substrates for both the firefly and renilla 
luciferase (Promega) using a LB96V microplate luminometer (Berthold Tech.). The expression of the firefly luciferase was recorded for the cells transfected with the various siRNA conjugates and was normalized against the Renilla expression. Shown in S_Figure 3 is the expression of firefly Luciferase relative to renilla Luciferase in HeLa cells treated with siRNA alone, lipofectamine-siRNA and SWNT-PL-PEG-SS-siRNA respectively. Consistent with our data for LaminA/C RNAi, the SWNT-SS-siRNA affords excellent silencing of the firefly Luciferase in HeLa cells. 


\section{Supplementary Figures}

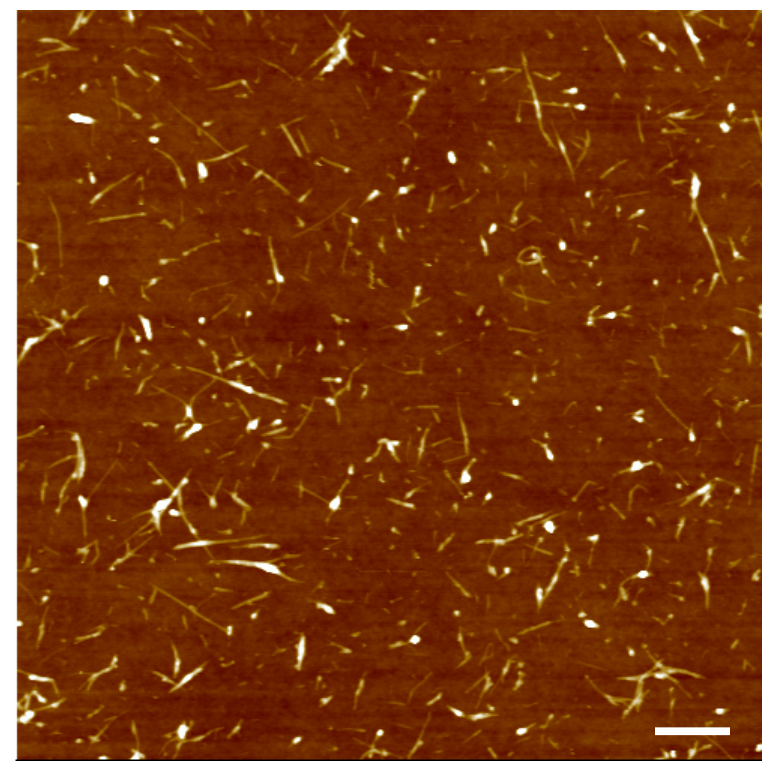

S_Figure 1. Atomic force microscopy (AFM) image of 1-DNA, revealing that most nanotubes were individualized or in small bundles with lengths ranging from 50-300 $\mathrm{nm}$. Scale bar $=300 \mathrm{~nm}$ 


\section{a}
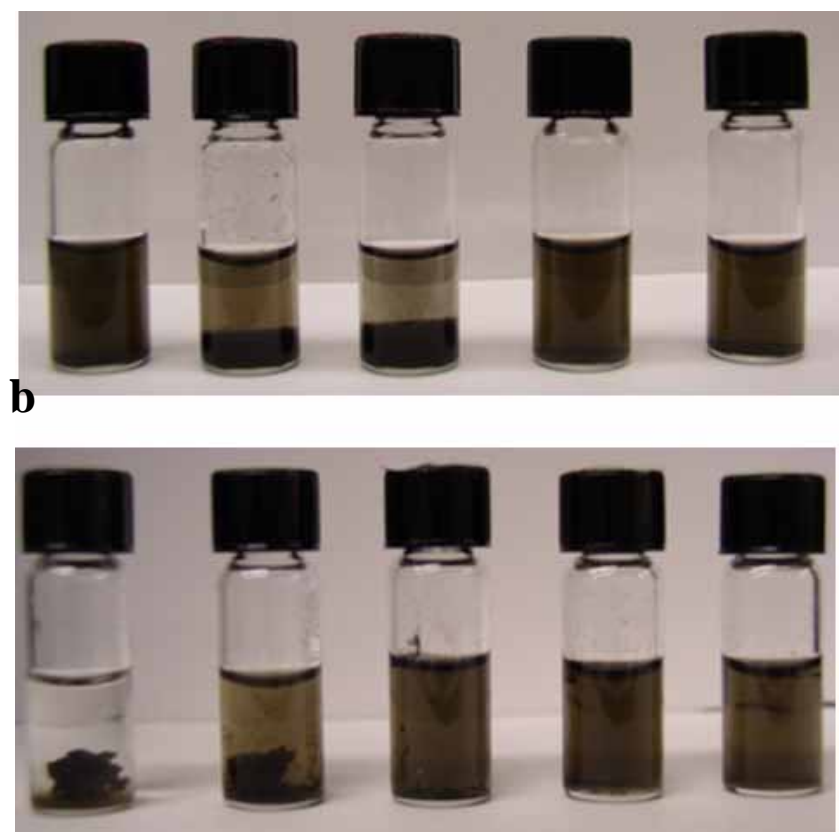

C

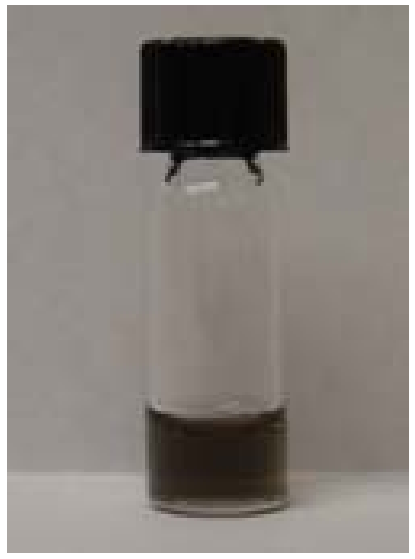

S_Figure 2. Photographs of different SWNT aqueous suspensions. SWNT functionalized with (i) tween-20 (ii) Gum Arabic (iii) Sodium dodecyl sulfate (SDS) and (iv) PL-PEG terminated with a methyl group and (v) PL-PEG with an amine group after (a) heating to $80{ }^{\circ} \mathrm{C}$ and (b) adding $0.2 \mathrm{M}$ phosphate buffer, $\mathrm{pH} 7.0$ respectively. The PL-PEG functionalized SWNTs in (iv) and (v) are stable after both treatments. (c) A solution of SWNT-PL-PEG-SS-siRNA (1-siRNA) showing sustained stability after standing for 2 months at $4^{\circ} \mathrm{C}$. 


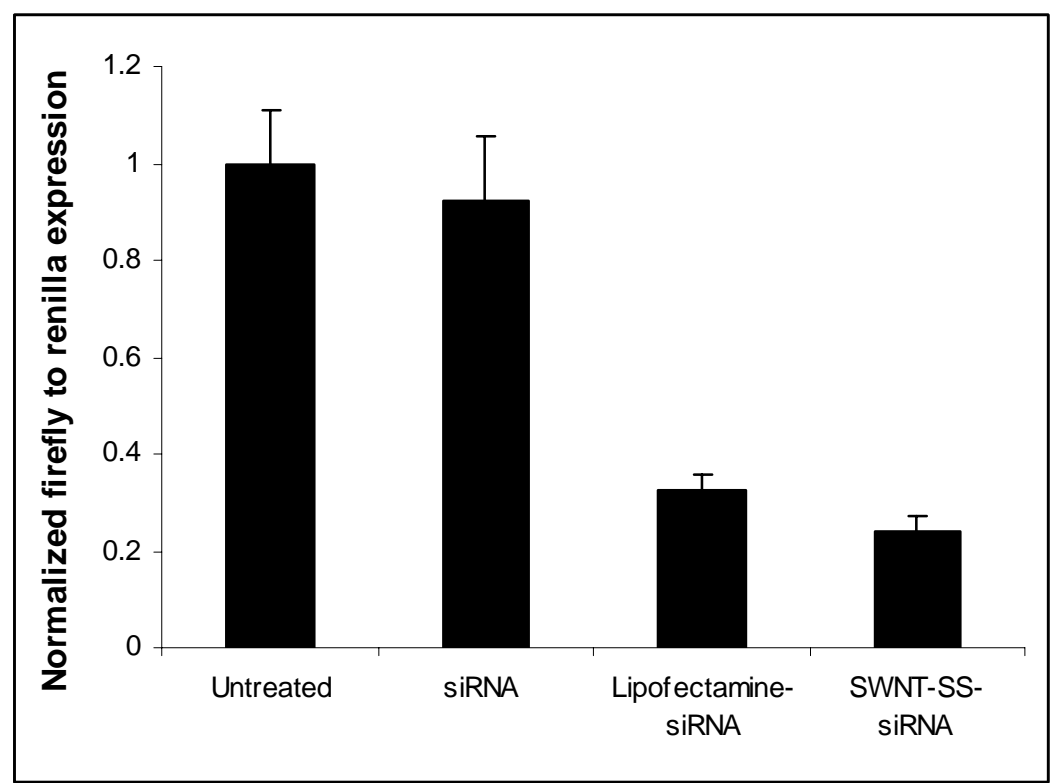

S_Figure 3. Firefly Luciferase expression assay after SiRNA treatment of HeLa cells ( 20,000 cells). The graph shows normalized expression level of firefly Luciferase relative to renilla luciferase after RNAi (specific to Firefly Luciferase) induction by siRNA alone, lipofectamine-siRNA and SWNT-PL-PEG-SS-siRNA respectively. The concentration of siRNA used for these studies was $500 \mathrm{nM}$. The Luciferase expression levels were obtained by measurement of the luminescence using a dual Luciferase assay kit. 